\title{
Redescrição de Litomosoides brasiliensis Almeida, 1936 (Nematoda: Filariidae) Parasito de Anoura caudifera (Chiroptera: Phyllostomidae)
}

\author{
Elizabeth D Mourão/ ${ }^{+}$, Leonardo dos S Avilla*, Herman Lent \\ Universidade Santa Úrsula, Instituto de Ciências Biológicas e Ambientais, Rua Fernando Ferrari 75, 22231-040 Rio de Janeiro, \\ RJ, Brasil *Museu Nacional, Universidade Federal do Rio de Janeiro, Rio de Janeiro, RJ, Brasil
}

Redescription of Litomosoides brasiliensis Almeida 1936 (Nematoda: Filariiidae) from a new host Anoura caudifera (Chiroptera: Phyllostomidae) - The study of the surface topography added details regarding the disposition of male caudal papillae, spicules and area rugosa apart from vulva and oral aperture. The occurrence of this nematode in the state of Amapá represents a new geographical distribution.

Palavras-chave: Litomosoides brasiliensis - Anoura caudifera - Nematoda - Chiroptera - Brasil

Anoura caudifera Geoffroy, 1818 é um morcego filostomídeo de pequeno porte ( 8 a $10 \mathrm{~g}$ ) que tem sua distribuição restrita às florestas úmidas da América do Sul, ao leste dos Andes (Eisenberg 1989).

Durante expedições científicas realizadas entre 1994 e 2000 em diferentes regiões do Brasil, espécimes de $A$. caudifera foram coletados e necropsiados e foram encontrados nematóides do gênero Litomosoides Chandler, 1931 na cavidade abdominal. Espécies desse gênero parasitam em sua maioria a cavidade abdominal de quirópteros; no entanto algumas espécies foram encontradas nas cavidades abdominal e torácica (pleura) de roedores (Rêgo 1961, Esslinger 1973).

Rêgo (1961) e Brant e Gardner (2000) citaram as seguintes espécies de Litomosoides em quirópteros do Brasil: Litomosoides guiterasi (Vigueras, 1934) em Glossophaga soricina soricina e Glossophaga sp.; L. brasilliensis Almeida, 1936 em Phyllostomidae, G. soricina e Carollia perspicillata; L. chandleri Esslinger, 1973 em Artibeus jamaicensis, Tadarida laticaudata e Phyllonycteris poeyi; L. leonilavazquezae Caballero \& Caballero, 1939 em G. soricina e L. molossi Esslinger, 1973 em Molossus major.

L. brasiliensis é agora redescrito através da microscopia eletrônica de varredura (MEV), em um novo hospedeiro e com distribuição geográfica ampliada.

\section{MATERIAIS E MÉTODOS}

Os morcegos foram capturados com redes de espera em três localidades: Vila Nova, Amapá (0³7’ N, 51ํ7' W), norte do Brasil (Projeto "Diversitas Neotropica”, Universidade Santa Úrsula, Muzeul di Historia Naturala Grigori Antipa); Carmo (21 ${ }^{\circ} 56^{\prime}$ S, 42³7’W), Rio de Janeiro, Su-

Com auxílio do CNPq, Faperj, Capes e USU

${ }^{+}$Autor de correspondência. Fax: +55-21-2551.6446. E-mail: e_mourao@hotmail.com

Recebido em 13 de junho de 2001

Aceito em 28 de janeiro de 2002 deste do Brasil e Bonito $\left(21^{\circ} 08^{\prime} S, 56^{\circ} 28^{\prime} \mathrm{W}\right)$ Mato Grosso do Sul, Oeste do Brasil (Projeto Biodiversidade do Quaternário do Brasil - Museu Nacional, UFRJ).

Os nematóides adultos foram coletados da cavidade abdominal, fixados em álcool 70\%, diafanizados em ácido acético $2 \%$ ou fenol e conservados em álcool $70 \%$. Foram desenhados em câmara clara no microscópio ZeissAxioscop. As medidas em micrômetros são apresentadas com os valores mínimo e máximo seguidos da média e do número de indivíduos medidos. Medidas de corpo inteiro foram obtidas somente a partir de espécimes machos pois as fêmeas estavam rompidas.

Para estudo em MEV os nematóides foram pós-fixados em solução de $\mathrm{O}_{\mathrm{S}} \mathrm{O}_{4} 1 \%$ em condição de escuridão, lavados em tampão fosfato $0,1 \mathrm{M}$, desidratados em série alcoólica, levados ao ponto crítico com $\mathrm{CO}_{2}$, metalizados e examinados ao microscópio JEOL JSM 8500.

\section{RESULTADOS}

Litomosoides brasiliensis Almeida, 1936

Sinonimia: Litomosoides carolliae Caballero y Caballero, 1944; Litomosoides caballeroi Gracia-Rodrigo, 1960

Hospedeiro: Anoura caudifera Geoffroy, 1818

Local de coleta: estados do Amapá, Rio de Janeiro e Mato Grosso do Sul, Brasil

Habitat: cavidade abdominal

Material depositado: CHIOC no 34.536 a-c; $34.537 \mathrm{a}-\mathrm{b}$

Descrição: corpo alongado transversalmente, 5000-5650 x 150-420 (5426 x 320)3, com cutícula estriada nos machos. Extremidade cefálica levemente arredondada. Boca terminal, ovalada com lábios pouco nítidos e papilas cefálicas subterminais. Cápsula bucal cilíndrica 13-23 (20) x 7-9 (8)8 nos machos e 13-27 (19) x 4-7 (6)5 nas fêmeas, com espessamentos quitinosos desiguais nas paredes. Esôfago 550-860 (720)8 nos machos e 530-710 (620)3 nas fêmeas (Figs. 1 a 3). Intestino retilíneo.

Machos com cauda fortemente espiralada apresentando a região ventral recoberta por numerosas fileiras de estrias cuticulares, sendo cada fileira composta por 26 a 41 estrias longitudinais de tamanhos distintos (Figs. 4-5). As estrias menores localizadas nas extremidades medem 3-4 enquanto as centrais alcançam 6-9 de comprimento. 

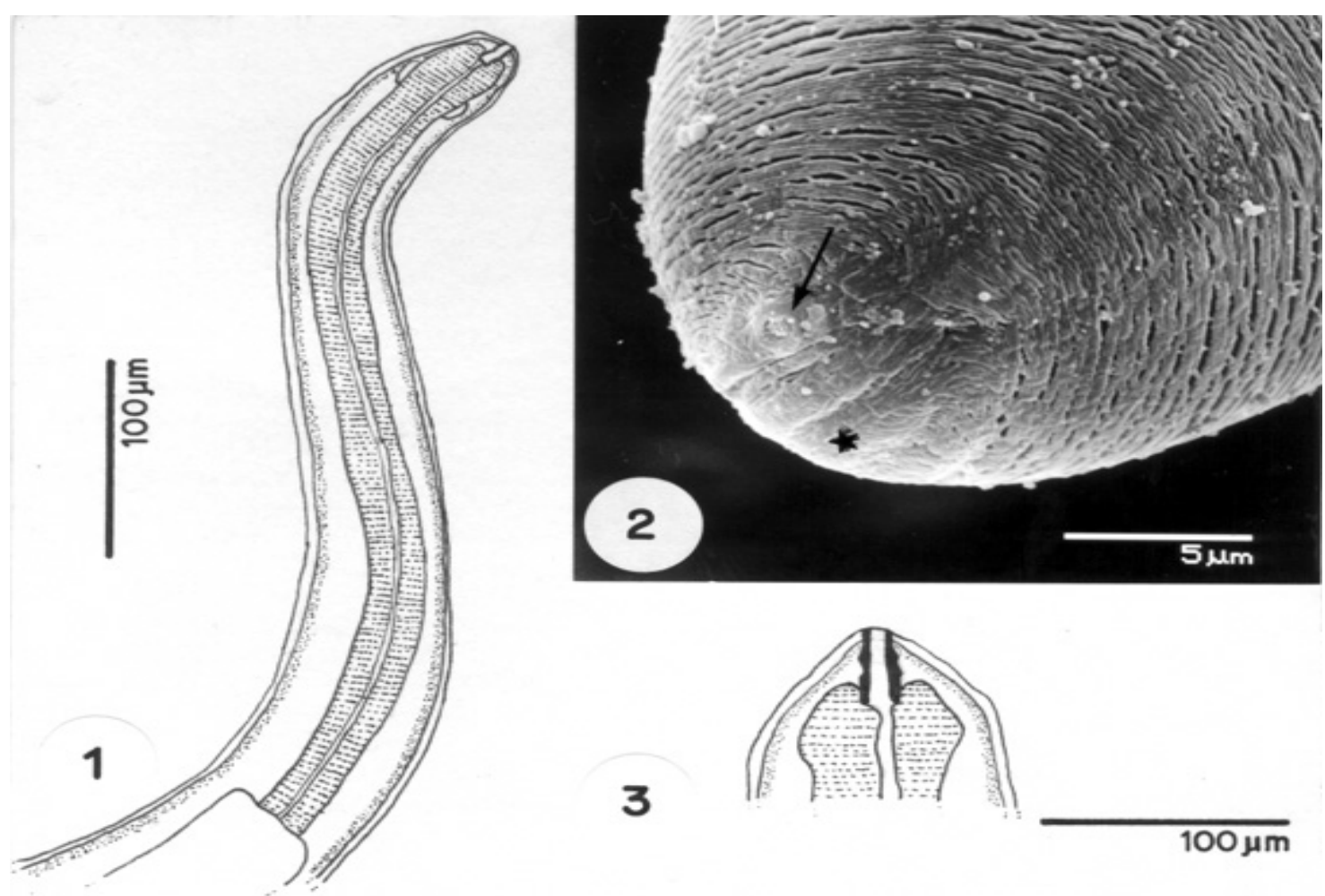

Litomosoides brasiliensis - Fig. 1: região anterior. Fig. 2: microscopia eletrônica de varredura com detalhe dos lábios (estrela) e papilas bucais (seta). Fig. 3: cápsula bucal

Possuem de quatro a cinco pares de papilas pós-cloacais ligeiramente alinhadas verticalmente (Fig. 6). Espículos desiguais e dissemelhantes parcialmente quitinizados, o maior 400-570 (530)8 com porções membranosas e o menor 120-200 (160)8 com ponta triangular característica (Figs. 7a-b). Na extremidade caudal aparecem quatro fasmídeos terminais (Fig. 8). Distância cloaca-cauda 140$280(170) 8$.

Fêmeas didelfas opistodelfas. Vulva de lábios pouco salientes sendo o inferior revestido por cutícula espessa a 1370-2230 (1800)3 da extremidade anterior, bem após o fim do esôfago (Figs. 9-10). Extremidade posterior do corpo retilínea afilando gradativamente até a cauda (Fig. 11). Distância ânus-cauda 150-460 (320)6. Microfilárias no útero com 27-92 (62)15 de comprimento.

\section{DISCUSSÃO}

Espécies de Litomosoides são encontradas exclusivamente em mamíferos das regiões Neoártica e Neotropical ocorrendo em sua maioria na América do Sul. No entanto as espécies que parasitam roedores e marsupiais não parasitam morcegos (Brant \& Gardner 2000).

As espécies de Litomosoides apresentam caracteres cefálicos reduzidos, forma da cabeça atenuada e ornamentações cuticulares encontradas em nematóides de vida livre, sendo essas reduções talvez uma adaptação ao tecido do hospedeiro ou adaptações evolutivas (Brant \& Gardner 2000). Bain et al. (1989) consideram a cápsula bucal reduzida com seus diferentes tipos de espessamentos nas laterais como o caracter morfológico mais importante para diferenciar espécies desse gênero.

Os espécimes estudados neste trabalho foram identificados como L. brasiliensis, sendo a cápsula bucal e a forma dos espículos maiores com partes membranosas e os menores com ponta triangular, características marcantes da espécie. Bain et al. (1989) colocam a espécie dentro do grupo carinii caracterizado pelo espículo direito esclerotizado até a extremidade distal, com capa terminal e lâmina do espículo esquerdo simples, sem grandes asas longitudinais membranosas.

L. brasiliensis foi descrito originalmente de um único exemplar macho com o espículo maior quebrado, coletado de Myotis sp. no estado de Mato Grosso, Brasil. Em 1961 a espécie foi redescrita por Rêgo a partir de material depositado na Coleção do Instituto Oswaldo Cruz, coletado em Phyllostomidae, Glossophaga soricina, Carollia perspicillata e Glossophaga sp. dos estados do Amazonas, Mato Grosso, Mato Grosso do Sul, Minas Gerais, Paraná, Rio de Janeiro e São Paulo. Na Venezuela foi referido em Myotis sp., Phyllostomus sp. e Carollia perspicillata (Diaz-Ungria, 1963).

Rêgo (1961) coloca L. carolliae e L. caballeroi em sinonímia com L. brasiliensis (Padilha e Barros-Araújo 1976).

Diaz-Ungria (1963) e Notarmicola et al. (2000) descreveram os pares de papilas caudais como sendo enfileirados, estando de acordo com o que foi agora revelado pela MEV. Diaz-Ungria (1963) relatou ainda a presen- 


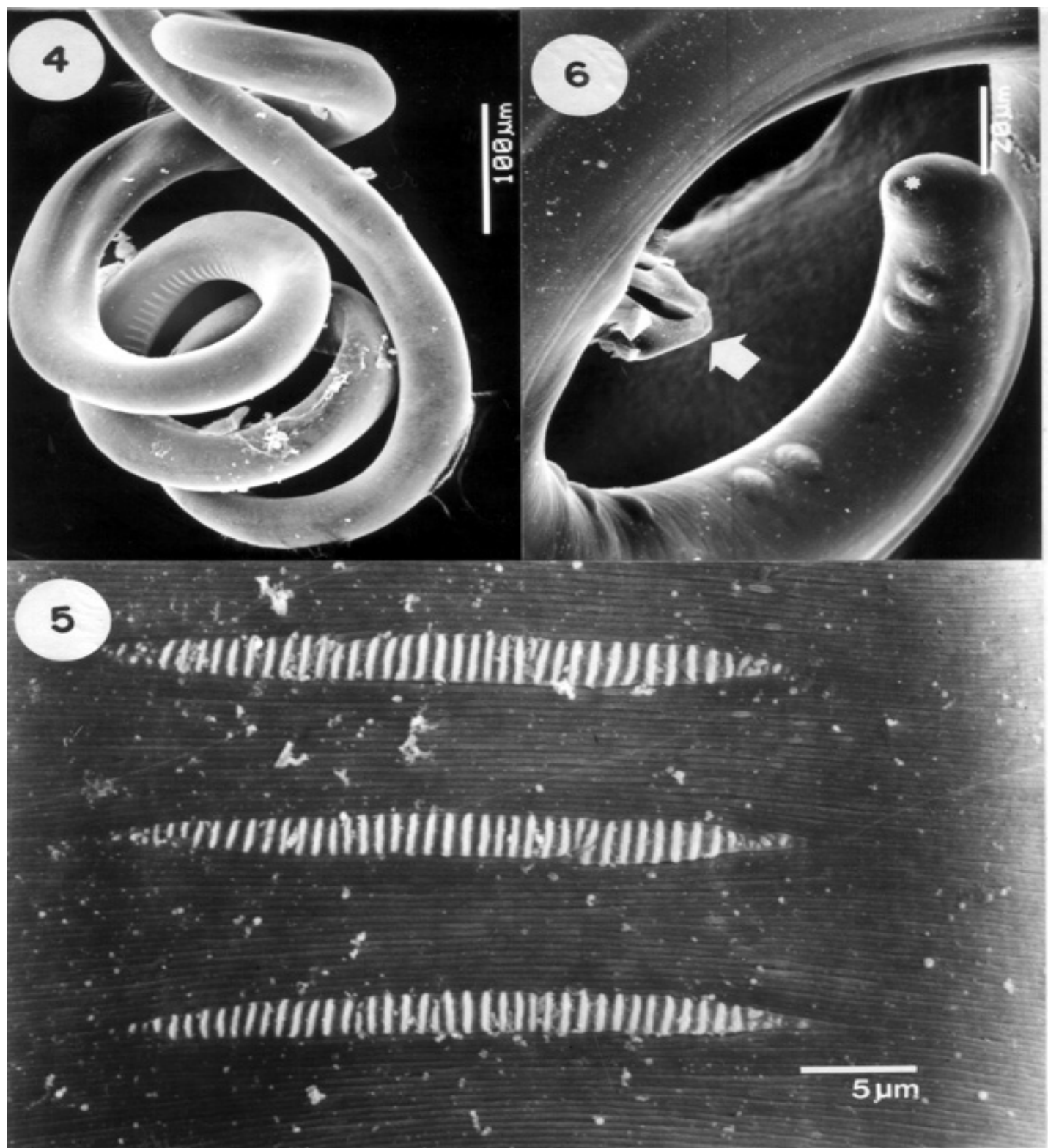

Macho de Litomosoides brasiliensis - Fig. 4: microscopia eletrônica de varredura (MEV) da cauda espiralada. Fig. 5: MEV da região ventral da cauda com detalhe das estrias. Fig. 6: MEV das papilas pós-cloacais mostrando a porção terminal do espículo direito (seta) e fasmídeos (asterisco).

ça de quatro pares de fasmídeos, sendo que tanto na MEV quanto na microscopia de luz apenas dois pares estão bem evidenciados. Notarmicola et al. (2000) descreveram ainda $L$. bonaerensis, do grupo carinii, de Muridae da Argentina, que apesar de ter o espículo direito semelhante ao de L. brasiliensis, com uma dilatação terminal, apresenta diferenças no tamanho dos espículos e disposição das papilas, além de parasitar um grupo distinto de hospedeiros.

Moraes Neto et al. $(1996,1997)$ descreveram L. silvai e L. chagasfilhoi, parasitas de roedor, pela MEV. Destas duas espécies apenas $L$. silvai pertence ao grupo carinii e apresenta as papilas da cauda do macho enfileiradas, área rugosa mostrando bandas com protuberâncias cuticulares discoidais assim como em L. brasiliensis. As duas espécies, $L$. silvai e $L$. brasiliensis, se diferenciam pela ponta do espículo direito, presença de fasmídeos e grupo de hospedeiros.

Novos dados morfológicos de L. brasiliensis são agora apresentados através da MEV que evidenciou a área rugosa da cauda do macho, a disposição das papilas caudais não arranjadas em pares, a presença de fasmídeos, além de detalhes dos lábios, das papilas labiais subterminais e da abertura bucal.

A ocorrência de L. brasiliensis no estado do Amapá, Brasil representa uma ampliação na distribuição geográfica deste filarídeo, sendo A. caudifera referido como um novo hospedeiro. 


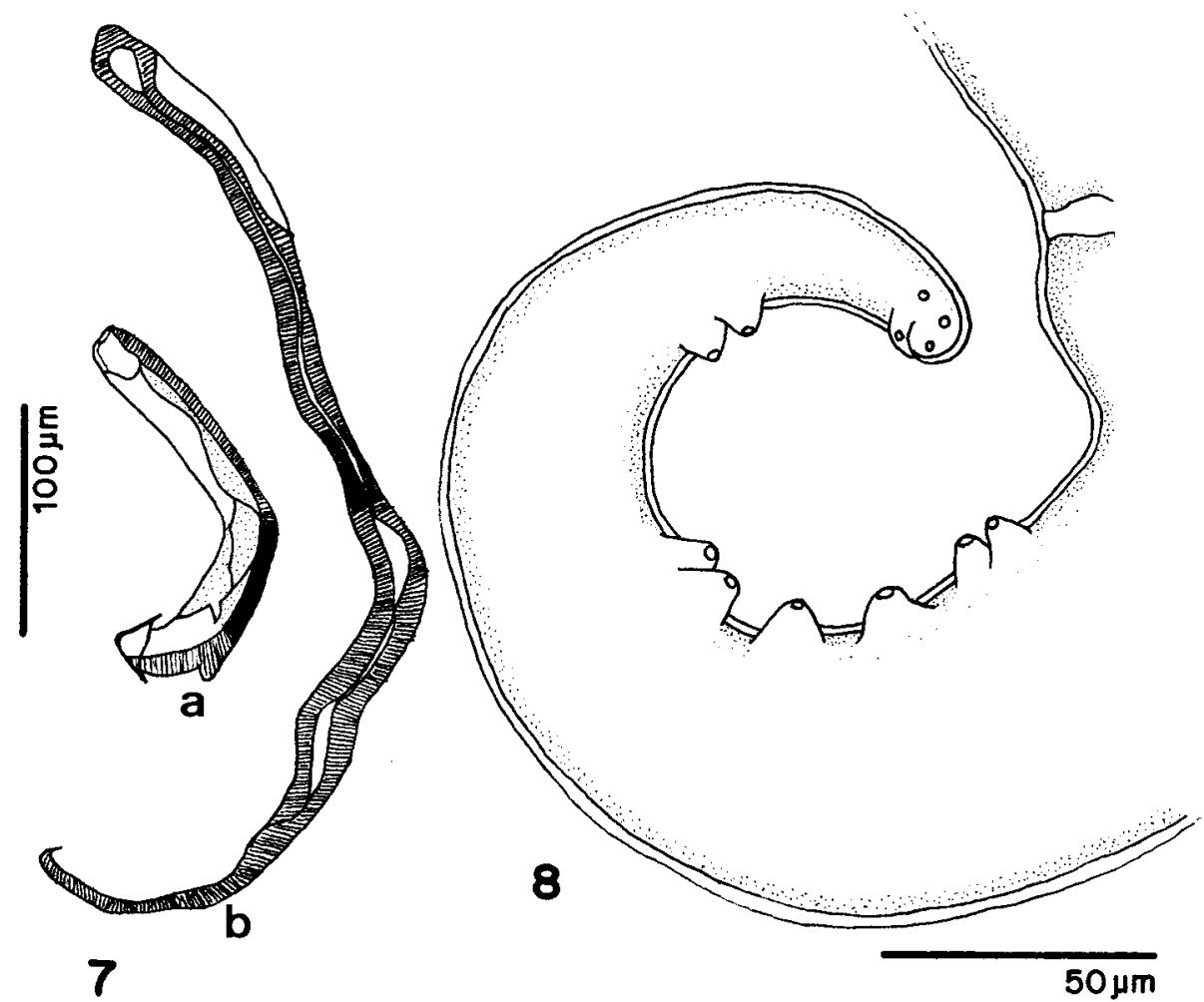

Macho de Litomosoides brasiliensis - Fig. 7a: espículo direito; b: espículo esquerdo. Fig. 8: cauda do macho mostrando as papilas póscloacais e os dois pares de fasmídeos terminais.
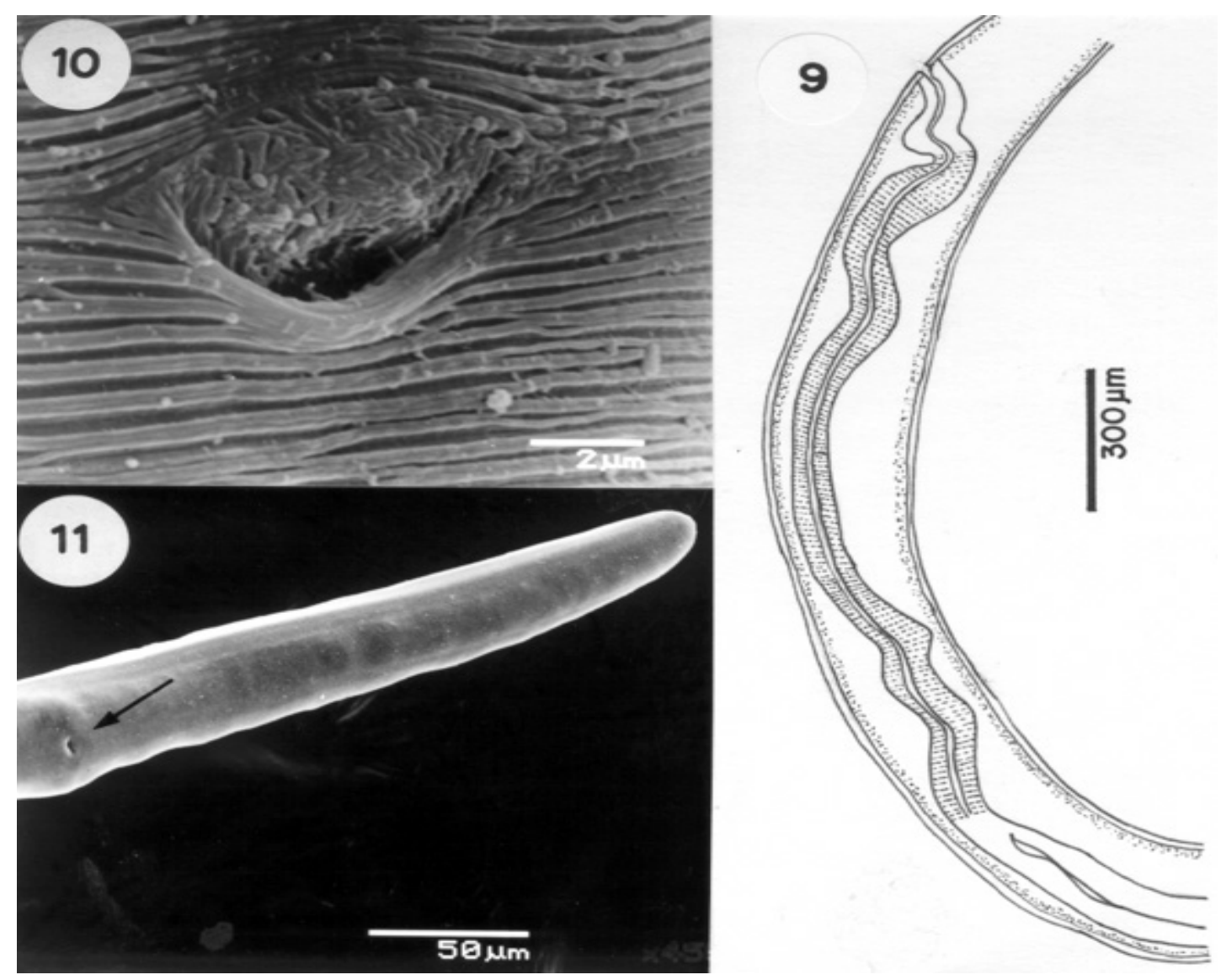

Fêmea de Litomosoides brasiliensis - Fig. 9: vulva. Fig. 10: microscopia eletrônica de varredura (MEV) da vulva mostrando o lábio inferior revestido por cutícula espessa. Fig. 11: MEV da cauda lisa com abertura anal (seta) 


\section{AGRADECIMENTOS}

A Dra. Claudia Portes Santos, Universidade Santa Úrsula pelos comentários e revisão do manuscrito. A Dra. Cláudia A Russo coordenadora do Projeto Biodiversidade do Quaternário do Brasil, Museu Nacional, Universidade Federal do Rio de Janeiro, pelo auxílio na obtenção dos quirópteros.

\section{REFERÊNCIAS BIBLIOGRÁFICAS}

Bain O, Petit G, Diagne M 1989. Étude de quelques Litomosoides parasites de Rongeurs; consequénces taxonomiques. Ann Parasit Hum et Com 64: 64-68.

Brant SV, Gardner SL 2000. Phylogeny of species of the genus Litomosoides (Nematoda: Onchocercidae): Evidence of rampant host switching. J Parasitol 86: 545-554.

Diaz-Ungria C 1963. Nématodes parasites, nouveaux ou intéressants, du Vénézuéla. Ann Parasit (Paris) 38: 893914.

Eisenberg JF 1989. Mammals of the Neotropics: Panama, Colombia, Venezuela, Guyana, Suriname, French Guiana, The University of Chicago Press, Chicago, 449 pp.

Esslinger JH 1973. The genus Litomosoides Chandler, 1931
(Filarioidea: Onchocercidae) in Colombian bats and rats. $J$ Parasitol 59: 225-246.

Moraes Neto AHA, Lanfredi RM, De Souza W 1996. Emended description of Litomosoides silvai (Nematoda: Filaroidea) of Akondon cursor (Rodentia: Muridae). J Parasitol 82: 988-991.

Moraes Neto AHA, Lanfredi RM, De Souza W 1997. Litomosoides chagasfilhoi sp. nov. (Nematoda: Filarioidea) parasitizinhy the abdominal cavity of Akodon cursor (Winge, 1887) (Rodentia: Muridae) from Brazil. Parasitol Res 83: 137-143.

Notarmicola J, Bain O, Navone GT 2000. Two new species of Litomosoides (Nematoda: Filarioidea) in Sigmodontines (Rodentia: Muridae) from Rio De La Plata Marshland, Argentina. J Parasitol 86: 1318-1325.

Padilha TN, Barros-Araújo JL 1976. Estudo sobre duas espécies do gênero Litomosoides Chandler, 1931, com novos aspectos biológicos (Nematoda: Filaroidea). Rev Bras Biol 36: 251256.

Rego AA 1961. Sobre algumas espécies do gênero Litomosoides Chandler, 1931 (Nematoda, Filarioidea). Mem Inst Oswaldo Cruz 59: 1-9. 
